\title{
The Effect of a Soccer Tournament on Baseline Psycho-Hormonal States of Collegiate Female Players
}

\author{
${ }^{1}$ Adele Broodryk ${ }^{(D)}{ }^{*}{ }^{1}$ Cindy Pienaar ${ }^{(D)},{ }^{1}$ Martinique Sparks \\ ${ }^{1}$ Physical Activity, Sport and Recreation Research Focus Area, Faculty of Health Sciences, North-West \\ University, Potchefstroom, South-Africa.
}

Submitted 03 February 2021; Accepted in final form 09 March 2021.

\begin{abstract}
Background. Anxiety plays a major role when it comes to sports performance, not only mentally, but physically as well. Objectives. The study aimed to examine the effect of a soccer tournament on baseline anxiety [Spielberger State-trait anxiety inventory questionnaire (STAI), mood [Incredible Short POMS questionnaire (ISP)] and cortisol (saliva sampling) states. Methods. Eight sportswomen's (age: $23.1 \pm 3.2$ y, playing experience: $10.6 \pm 5.6$ y) data were collected an hour after waking, two weeks prior to, and each morning during, a five-day tournament. Results. Overall, a small relationship was seen between true cortisol values and presence of state-anxiety $(\mathrm{r}=0.3, \mathrm{P}=<0.05)$. On mornings prior to a match lost, a significant relationship was seen between cortisol and the current- $(r=0.7, \mathrm{P}=0.005)$ and total trait-anxiety scores $(r=0.7, \mathrm{P}=0.008)$. Following multiple regression analysis, the TAI questionnaire was demonstrated to be adequate to predict possible cortisol surges $\left(\mathrm{r}^{2}=0.3, \mathrm{P}=0.04\right)$. Measuring the innate anxiety characteristic can be a positive measure to anticipate both psychological (presence of anxiety, $\mathrm{r}^{2}=0.88, \mathrm{P}=0.001$ ) and physiological (cortisol surges, $\mathrm{r}^{2}=0.4, \mathrm{P}=0.008$ ) stress. Conclusion. Therefore, an anxiety questionnaire might provide sensitive information regarding the unconscious physiological and psychological stress plausibly altering performance. It is recommended that a player's state of anxiety (innate and current) be measured prior to a competition to adopt a strategy to overcome its negative consequences.
\end{abstract}

KEYWORDS: Soccer, Hydrocortisone, Anxiety, Psychological Stress, Physiological Stress.

\section{INTRODUCTION}

Playing a tournament can place a tremendous load on the body, not only physically but also physiologically and psychologically. A sport tournament usually consists of a few competitions played within a short period, leaving minimal time for optimal recovery and preparation for the next event (1). One particularly taxing tournament format, commonly played at collegiate level, is a week-long either round-robin or knockout contest. In these cases, players can play up to 5 or 6 matches within 4 or 5 days, even playing multiple matches on a single day. Emphasizing the need to adequately prepare the players at the physical, physiological and psychological extent.
With the popularity of female soccer increasing, and thereby providing more opportunities to train and compete in a professional setting, it has become necessary to evaluate the effects of a tournament on the psycho-hormonal states in order to adequately prepare them for the demands of the game $(2,3)$. In a recent study, it was reported that collegiatelevel female soccer players cover approximately $5800 \mathrm{~m}$ during a game, with midfielders covering the greatest distance (4). In addition to this, $13 \%$ of the total match time was spent in the highintensity-heart-rate zone, with defenders spending the greatest amount of time in this zone (13.3\%) (4). Taken collectively, the physical

* Corresponding Author:

Adele Broodryk, Ph.D.

E-mail: adele.broodryk@nwu.ac.za 
demands of a single match, together with the accumulating fatigue due to multiple matches and the stress accompanying the tournament, can lead to various physiological and psychological instabilities (1) which might alter performance (5). One key factor that influences performance is the pressure for success, which usually increases the experience of anxiety (5).

Anxiety is a motivational and emotional state (6) that usually arises before a competition, with players experiencing feelings of nervousness and tension as well as thinking and worrying about the upcoming event (7). However, a state of anxiety (the immediate response to a stressor) should be discriminated from trait-anxiety (a general tendency to respond with anxiety, which is a normal characteristic of an individual) (5). An individual who exhibits higher levels of trait anxiety tends to have higher state anxiety, thereby being at risk to experience reduced performance as explained by the inverted-U hypothesis (8). Man and colleagues (9) conveyed how players perform exceptionally during training and minor competitions, whereas performance diminished during important competitions, concluding that this might be due to anxiety (10). This might be due to players worrying excessively about the threat or objective, shifting their attentional resources either externally (threatening taskirrelevant distractors) or internally (experiencing worrisome thoughts) (6). Competitive anxiety can arise in two respects, cognitive anxiety, which is the mental component caused by negative expectations, whereas somatic anxiety refers to the affective and physiological elements developed as a result of the autonomic arousal (11). One physiological consequence is an increase in cortisol secretion (12) as a bidirectional relationship exists between cortisol and negative mental states (13).

Cortisol is an important glucocorticoid in the body (14), being involved in various physiological and psychological processes (1, 15). In this regard, cortisol plays a vital role in providing fuel for the body by increasing blood glucose levels as well as aiding in the effects of catecholamine and sympathetic activation (16). An over-secretion of cortisol, however, can be catabolic as it can inhibit the inflammatory process and immunity by reducing protein synthesis and increasing protein degradation (1). It is widely known that an increase in cortisol secretion takes place during a competition (refer to the review by Casto \& Edwards (17)), though most studies compared post- to pre-competition responses and not compare them with baseline values. This is of importance as anticipation can lead to an unexpected increase in cortisol, thereby not displaying the true cortisol basis. Studies reporting baseline values (18-21) reported higher cortisol values prior to a competition, though baseline samples were collected 10-30 min prior to the start of the warm-up. This again can be expected as the time leading up to a competition can be stressful, resulting in heightened cortisol secretion (17) and the experience of negative mental states (22). Examining baseline cortisol responses is vital not only to indicate unconscious stress and anticipation, but also for performance as an inverse relationship exist between various hormones (especially androgens such as testosterone and cortisol), psychological states and match outcome (23). The higher the baseline cortisol value, the less testosterone increases, which might result in reduced feelings of dominance, power, focus and motivation for success $(15,24)$. This in turn can reduce the likelihood of a winning outcome as previous studies reported on the correlation between high baseline testosterone levels and victory, compared to high cortisol and a defeat $(17,24)$.

Psychologically, hormonal fluctuations influence either the sensory system, integrators or the effector system (13). High cortisol levels have been associated with negative mood states such as anger, cognitive anxiety, depression and fatigue among others $(1,25-27)$. According to Smyth (12), there is a direct relationship between cortisol and mood, with low cortisol levels associated with positive mood states and vice versa. Therefore, it is plausible that mood can mediate the relationship between cortisol and stress (12), and thereby reduce the negative consequences observed due to over-secretion of cortisol. In addition to this, identifying participants prone to stress and whether a relationship exists between these states and their cortisol responses can be useful for implementing strategies to overcome negative mental states and thereby enhance their performance. 
Though an abundance of literature is available on the effects of competition on cortisol and mood states, there is little on the effect of a tournament on the baseline psycho-hormonal states. Moreover, studies including baseline samples vary widely regarding the sample collection times. Some studies reported baseline values taken $30 \mathrm{~min}$ before the competition, whereas others collected baseline samples a few days or weeks beforehand. Taking measurements too close to a competition can result in false baseline values, as anticipating the competition can stimulate cortisol secretion and engender negative mood states $(2,17,28)$. Unfortunately, the measurement of cortisol is time consuming and expensive, implying the need for other reliable ways to indicate the underlying physiological state of the athlete (2).

Though some studies were found indicating a relationship between cortisol and mental states, only a few evaluated whether a psychological questionnaire can be used to forecast the underlying stress, and whether this would correspond to the actual performances observed. To add to the scarce literature, this study evaluated the effect of a week-long tournament on baseline cortisol and psychological states taken two weeks prior to, as well as every morning upon waking during a tournament on collegiate female soccer players. Therefore, the aim of the current study was to identify the magnitude of the relationship between cortisol and psychological states and whether the application of a psychological questionnaire can aid in determining underlying physiological states. The results and the application thereof, can be construed as valuable for the coaching staff as it signifies how a psychological questionnaire is a simple yet effective way to determine causal physiological and psychological stress which alters performance.

\section{MATERIALS AND METHODS}

A repeated measure, quantitative research design with convenience sampling was implemented for the purpose of this study. Baseline measurements taken two weeks in advance, and during the period of a six-match tournament. Weather conditions recorded for the period ranged from $23-29^{\circ} \mathrm{C}$ (afternoon) and $7-14^{\circ} \mathrm{C}$ (morning), with average relative humidity at $28-49 \%$.
Participants. Eight healthy collegiate female soccer players $(23.1 \pm 3.2$ years; $158.9 \pm 6.4 \mathrm{~cm}$; $54.7 \pm 4.2 \mathrm{~kg}$, playing experience: $10.6 \pm 5.6$ years) from a tertiary education institution volunteered to participate in the study. Ethical approval (NWU-00055-15-A1) and permission from the tournament organisers and coaches were obtained before data collection. Subjects reported normal menstrual cycle phases with none taking oral contraceptives or prescribed medication and none in their menstrual phase at the time of testing. Only subjects playing the entire match and remaining free of sickness and injury were included in the study.

Methodology. Data were collected on two occasions: baseline data were collected two weeks before the tournament in the morning, and then every morning over a week-long tournament. The tournament comprised of six matches, four pools played in a knockout format with certain matches vital for play-off rankings to succeed to the final (2).

On the evening preceding the start of testing, all procedures were explained, and players completed the demographic and personal questionnaires. They were advised to obtain a good night's rest (at least 8 hours' sleep) and fast for at least an hour before the first data were collected (a precise waking-time was specified for every evening). Upon waking on the first day, their body mass (BFW Platform scale, Adam Equipment, UK) and stature (Harpenden portable stadiometer, Holtain Ltd., UK) were recorded. An hour after waking, a saliva sample and completed STAI and ISP questionnaires were collected. This regime was conducted two weeks prior during baseline measurement, at the onset and then over five consecutive days during the six matches of the tournament, between 6 am and 7 am every morning.

Saliva Sampling. Salivary samples were collected for the assessment of cortisol by using the passive drool test (29). Samples were stored in a freezer $\left(-20 \pm 1^{\circ} \mathrm{C}\right)$ until analysed by a professional laboratory. The cortisol content from $20 \mu \mathrm{l}$ saliva samples were determined by using a cortisol saliva luminescence immunoassay. The samples were transferred into a Berthold Luminometer to calculate the average relative luminescence units, which were plotted against the concentration to determine the exact value. 
There is both a linear and non-linear correlation coefficient ( $r=0.8-1.0)$ between salivary and serum cortisol values based on this method. This specific biochemical analysis has an intra-CV ranging between $0.4-1.7 \%$ and inter- $\mathrm{CV}$ between $0.8-1.8 \%$ (30). To minimize the effect of circadian rhythm, samples were collected one hour after waking, with the subjects also fasting for at least an hour, this is due to both the waking response and a meal to increase cortisol secretion immensely. (31) Owing to the diurnal rhythm of cortisol in the body, the collection times were logged and samples measured based on normal variations throughout a day (30). All cortisol measurements were standardized according to their collection time with each subject serving as her own control (32).

Sport Psychological Questionnaires. Incredibly Short Profile of Mood States (ISP), The ISP derived from the original Profile of Mood states questionnaire (33) compiled by Dean and colleagues (34), was completed upon waking. Correlations ranging from $r=0.67-0.82$ between the two questionnaires have been previously reported (34). The ISP consist of six questions that measure the six mood subscales (anger, depression, tension, confusion, fatigue and vigour). The subjects rated the questions on a 5 point Likert scale: 1 ('not at all'), 2 ('a little'), 3 ('moderately'), 4 ('quite a bit') and 5 ('extremely'). Individual scores for each subscale was the rating indicated per question and the total mood disturbances (TMD) score calculated by adding the negative subscales and subtracting the positive scale.

State-Trait Anxiety Inventory (STAI), The State-Trait Anxiety Inventory (STAI) was used to assess the subjects' perceived state anxiety levels upon waking (35). The STAI is a 40-item, selfevaluation questionnaire which includes separate measures to determine a subject's state and trait anxiety, respectively. The State-Anxiety scale consists of 20 statements that evaluate how players feel "right now, at this moment" on a 4-point Likert scale: 1 (not at all), 2 (somewhat), 3 (moderate so) and 4 (very much so). The Traitanxiety scale also consists of 20 statements that assess how people "generally feel" on a 4-point Likert scale: 1 (almost never), 2 (sometimes), 3 (often), and 4 (almost always). Four indicates the presence of high anxiety levels where 1 indicates the absence of high anxiety levels Anxiety levels were determined by calculating the sum of the different scores. The range of scores can vary from 20-80; the higher the score, the greater the anxiety perceived (35). Internal consistency reliability for state anxiety $(\mathrm{r}=0.91)$ has been reported in previous research (36).

Statistical Analyses. IBM SPSS Statistics software (v. 25.0.0.0) was used for data analyses. Descriptive statistics (means, maximum, minimum, and standard deviation values) were calculated at every time point for each variable. Afterwards, linear mixed model analyses were completed to investigate time point differences with an autoregressive 1 covariance structure. The time points were entered as the fixed effects and players as the subjects. Following this, the cortisol values were graded according to the subjects' waking and sample collection times (29, $30,32)$, after which it was ranked into three categories (low, average/neutral or high) (32). Spearman's rank correlation rho was used to determine the relationship between all variables (cortisol, TMD, SAI and match outcome). A Fisher's $r$ to $z$ transformation was calculated to determine the $90 \%$ confidence interval (CI) from the correlation coefficient. To examine the extent to which the psychological questionnaires (ISP and STAI) predicted underlying cortisol responses, a series of linear regression analyses were performed. The psychological questionnaires and their substates were entered as independent variables in separate models, both with and without adjustments for the covariate's true cortisol responses and trait anxiety. The cortisol responses were adjusted according to the wakening and collection time and ranked in its respective three categories as previously mentioned as it follows a circadian rhythm. Trait anxiety were entered as "individuals" with a proneness to experience situations as "threats", which might as a result, demonstrate high state anxiety levels.

The level of significance was set at $\mathrm{P} \leq 0.05$. The strength of the correlation was categorized from $\leq 0.01$ (trivial) to $\geq 1$ (perfect). Cohen's effect sizes (d) were interpreted as large ( $\geq 0.8)$, moderate $(\geq 0.5)$ and small ( $\geq 0.2)$ (37). In each case, magnitudes of standardised effects $\pm 95 \%$ confidence interval $(\mathrm{d} \pm 95 \% \mathrm{CI})$ were assessed as $0-0.2$ trivial, $0.2-0.6$ small, $0.6-1.2$ moderate, 
1.2-2.0 large, and > 2.0 very large (38). Effect sizes with wide confidence intervals that overlap categorical classifications of effect have been downgraded as "unclear".

\section{RESULTS}

Cortisol Results. As seen from Fig. 1, a significant higher cortisol value was collected at day $1(\mathrm{~d}=0.9, \mathrm{CI}=0.2$ to 1.6$)$ and $3(\mathrm{P}=0.01, \mathrm{~d}$ $=1.4, \mathrm{CI}=0.4-2.5)$ compared to baseline values.
Day 3 also demonstrated a large effect, compared to day $5(\mathrm{~d}=0.9, \mathrm{CI}=-0.2$ to 1.8$)$, though classified as unclear. This was further observed in the cortisol rankings, as day 3 had a large difference compared to baseline $(\mathrm{d}=1.2, \mathrm{CI}=$ $0.1-2.2)$ and day $5(\mathrm{~d}=1.6, \mathrm{CI}=0.4-2.6)$. A large correlation was observed between the precise cortisol values and its respective rankings $(\mathrm{r} \geq 0.9$, $\mathrm{P}<0.001)$.
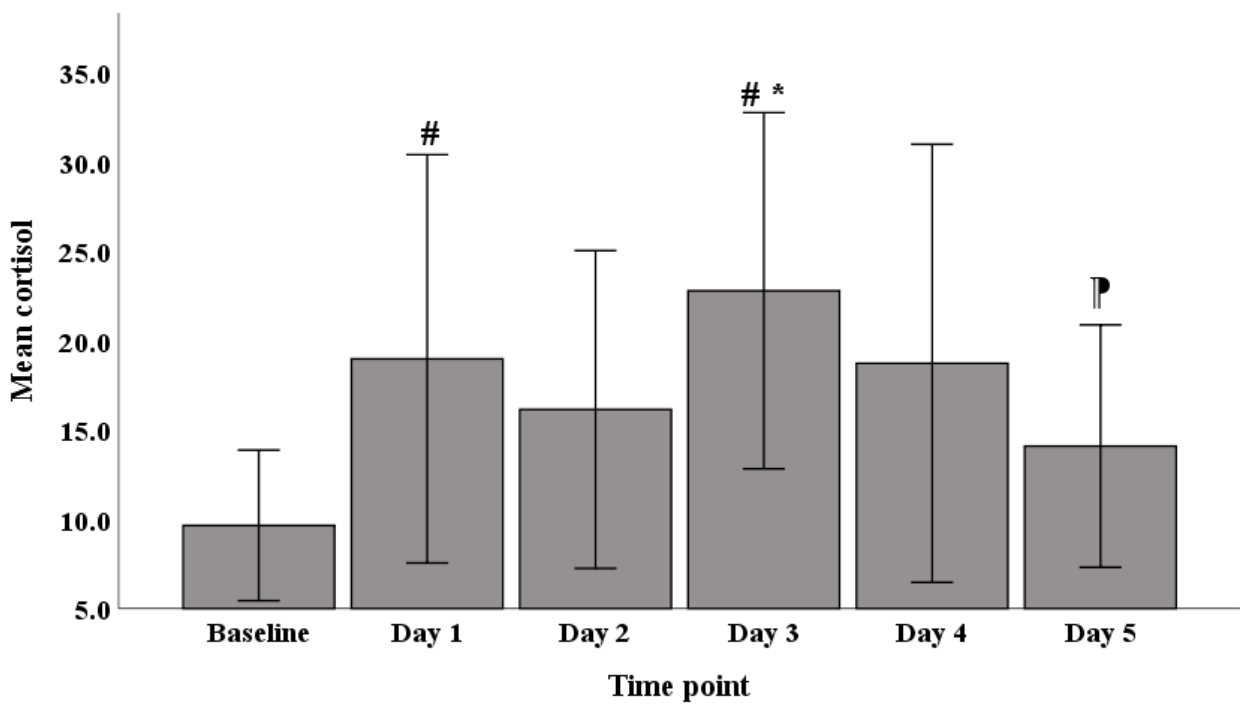

Error Bars: $95 \%$ CI

Figure 1. Mean Cortisol Values Over the Various Time Points.

\# Large difference compared to baseline $(\mathrm{d}>0.9)$; ${ }^{*}$ Significant difference compared to baseline $(\mathrm{P}<0.05)$; $\mathbb{P}$ Significant and large difference compared to day $3(P<0.05)$.

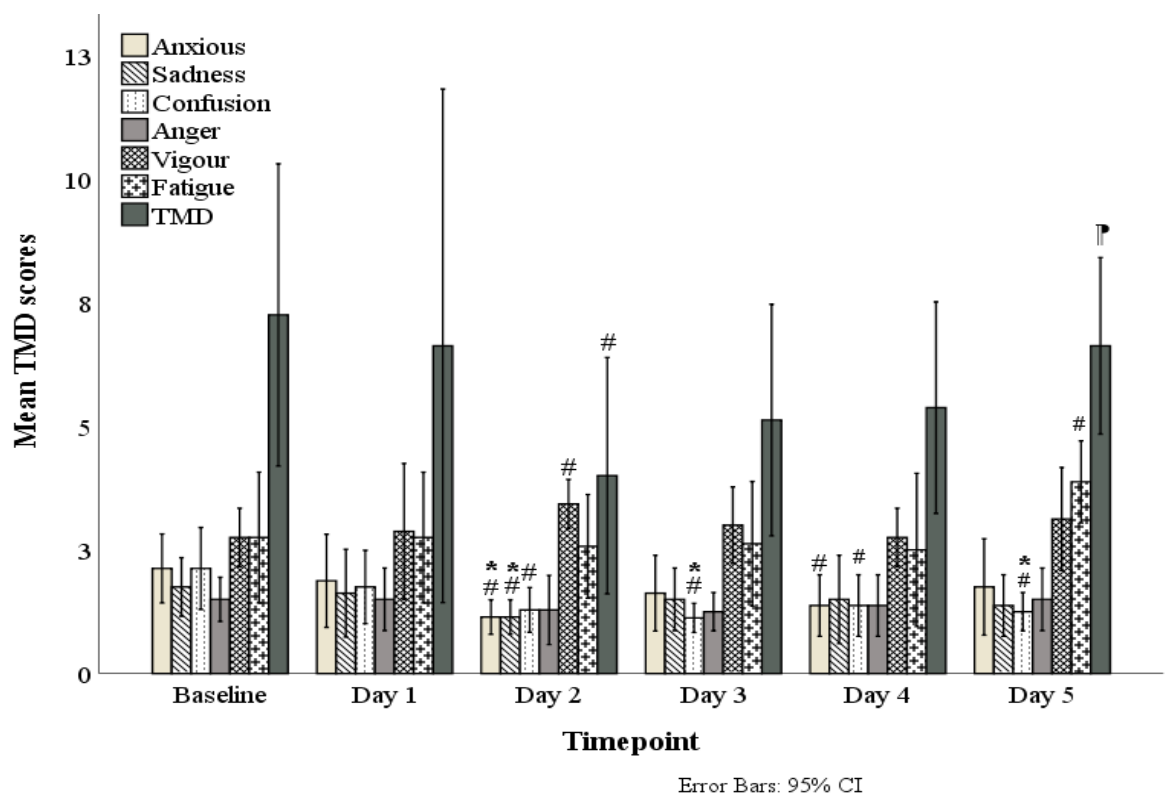

Figure 2. Mean TMD Responses Over the Various Time Points.

\# Large effect compared to baseline $(\mathbf{d}>0.9)$; ${ }^{*}$ Significant difference compared to baseline $(\mathbf{P}<0.05)$; $\mathbb{P}^{\mathbb{P}}$ Significant and large difference compared to day $2(P<0.05)$ 
Psychological Results. A significant difference was noted within several of the ISPsubscales over the various timepoints. In this regard, baseline measurements demonstrated significantly higher values in ISP-anxiety compared to day $2(\mathrm{P}=0.01, \mathrm{~d}=1.4, \mathrm{CI}=0.3$ 2.6) and day $4(\mathrm{~d}=0.9, \mathrm{CI}=-0.1-1.9)$; ISPsadness compared to day $2(\mathrm{P}=0.05, \mathrm{~d}=1.1, \mathrm{CI}$ $=-0.03-2.1)$; ISP-confusion compared to all the competition days $(\mathrm{P}>0.02, \mathrm{~d}>0.9)$ and ISPvigour compared to day $2(\mathrm{~d}=1.0, \mathrm{CI}=-0.1-2.0)$. Day 5's ISP-fatigue results demonstrated a large difference compared to baseline $(\mathrm{d}=0.9, \mathrm{CI}=$ $0.2-1.8$ ), though can be classified as unclear. Day 2 demonstrated the lowest overall TMD scores, being significantly lower than baseline $(d=1.0$, $\mathrm{CI}=-0.1-2.0)$ and day $5(\mathrm{P}=0.05, \mathrm{~d}=1.1, \mathrm{CI}=$ -0.04-2.1). In addition to this, day 2 also demonstrated significant lower scores compared to baseline for the state-subscale $(\mathrm{d}=0.9, \mathrm{CI}=$ 0.2-1.9) and trait-subscale $(\mathrm{P}=0.04, \mathrm{~d}=1.2, \mathrm{CI}=$ $0.05-2.2)$. Over the course of the tournament, anxiety slightly increased, though not significantly. Throughout the testing period a relationship was observed between trait- and state anxiety $(\mathrm{r}=0.77 \mathrm{P}=0.001)$, with the linear regression demonstrating a value of $\mathrm{r}^{2}=0.88(\mathrm{P}=$ 0.001 ), predicting a possible $85 \%$ of the state anxiety experienced upon waking.

Psycho-Hormonal Relationships. Overall, a small relationship was observed between cortisol and current state-anxiety $(\mathrm{r}=0.3, \mathrm{P}=0.04)$, as well as between the cortisol ranking values and present state-anxiety $(\mathrm{r}=0.33, \mathrm{P}=0.02)$. Of significance is the correlation observed prior to a defeat between the true cortisol values and current trait-anxiety $(\mathrm{r}=0.69, \mathrm{P}=0.005)$ as well as the total trait-anxiety scores $(\mathrm{r}=0.66, \mathrm{P}=0.008)$.

Table 1. Correlations among Cortisol and Psychological States at Baseline Overall, and Prior to a Victory or Defeat

\begin{tabular}{|c|c|c|c|c|c|c|c|}
\hline \multirow[t]{2}{*}{$\mathbf{r}$} & Cortisol & Cortisol Ranking & TMD & SAI Present & SAI Total & TAI Present & TAI Total \\
\hline & \multicolumn{7}{|c|}{ Victory } \\
\hline \multicolumn{8}{|l|}{ Defeat } \\
\hline Cortisol & - & $0.9 * *$ & 0.2 & 0.36 & 0.18 & 0.26 & 0.24 \\
\hline Cortisol rank & $0.88 * *$ & - & 0.34 & 0.36 & 0.21 & 0.16 & 0.13 \\
\hline TMD & 0.21 & 0.28 & - & $0.78 * *$ & $0.7 * *$ & 0.38 & 0.41 \\
\hline SAI present & 0.49 & $0.51 *$ & 0.43 & - & $0.87 * *$ & $0.64 *$ & $0.68 * *$ \\
\hline SAI total & 0.41 & 0.41 & $0.6^{*}$ & $0.94 * *$ & - & $0.7 * *$ & $0.68 * *$ \\
\hline TAI present & $0.69 * *$ & $0.71 * *$ & 0.42 & $0.86^{* *}$ & $0.83 * *$ & - & $0.95 * *$ \\
\hline TAI total & $0.66 * *$ & $0.68 * *$ & 0.51 & $0.8 * *$ & $0.86^{* *}$ & $0.91 * *$ & - \\
\hline \multicolumn{8}{|l|}{ Overall } \\
\hline Cortisol & - & $0.85 * *$ & 0.2 & $0.3^{*}$ & 0.19 & 0.25 & 0.19 \\
\hline Cortisol rank & & - & 0.3 & $0.33 *$ & 0.2 & 0.26 & 0.16 \\
\hline TMD & & & - & 0.5 & 0.6 & 0.4 & 0.5 \\
\hline SAI present & & & & - & $0.84 * *$ & $0.77 * *$ & $0.69 * *$ \\
\hline SAI total & & & & & - & $0.66^{* *}$ & $0.78 * *$ \\
\hline TAI present & & & & & & - & $0.81 * *$ \\
\hline TAI total & & & & & & & - \\
\hline
\end{tabular}

Table 2. Set of All Predictor Components to Cortisol Responses Prior to a Defeat.

\begin{tabular}{lcccccccc}
\hline Component & $\mathbf{r}$ & $\mathbf{r}^{\mathbf{2}}$ & $\mathbf{B}$ & St. Error & $\boldsymbol{\beta}$ & $\mathbf{t}$ & $\mathbf{p}$ \\
\hline Trait-anxiety present & 0.66 & 0.43 & 1.36 & 0.43 & 0.66 & 3.16 & 0.008 \\
Trait-anxiety total & 0.54 & 0.29 & 0.7 & 0.3 & 0.54 & 2.29 & 0.04 \\
\hline
\end{tabular}

This was further computed into a multiple linear regression model, and after adjusting for trait-anxiety, demonstrated that the TAI questionnaire can be implemented to predict both the true cortisol values, especially prior to a losing outcome $\left(r^{2}=0.54, P=0.04\right)$ with $30 \%$ explained by this questionnaire. In addition to this, the traitanxiety present subscale also predicted the true cortisol values prior to a losing outcome $\left(\mathrm{r}^{2}=\right.$ $0.66, \mathrm{P}=0.008$ ), with $43 \%$ explained thereby (see Table 2).

\section{DISCUSSION}

The current study, the first of its kind, evaluated the effect of a soccer tournament on baseline cortisol and psychological states of 
female soccer players in South Africa. The most significant finding was the value of implementing an anxiety questionnaire to predict underlying cortisol levels. This is desirable as an oversecretion of cortisol can have various negative effects. Our results also confirmed the stress that players experience during a tournament, as indicated by significantly higher cortisol values upon waking during the tournament compared to two weeks beforehand. Furthermore, it was observed how players with a high level of traitanxiety (thereby being prone to anxiety) also demonstrated a high state of anxiety and cortisol secretion, indicating a higher stress-response to daily disturbances (39). It is therefore recommended that the coaching staff determine the players' proneness to anxiety by evaluating their trait anxiety, and subsequently introducing them to coping strategies to reduce the physiological and psychological negative consequences of amplified anxiety as postulated by the inverted U-hypothesis (8).

A progressive increase in cortisol readings took place during the tournament compared to baseline, which is similar to previous research $(25,40)$. These values peaked at day three and then slightly decreased, though remained still higher than baseline values. This progressive increase might be an indication of fatigue taking place over the course of the tournament, as studies have demonstrated a direct relationship between cortisol and perceived exertion $(32,41)$. This likely fatigue was not only noticed for the cortisol results, but the internal and external player loads (refer to Broodryk et al. (2) for the exact values) also indicated a progressive increase in distance covered from the first to third match day, after which it decreased. The volume of low-intensity activities executed also decreased over the course of the tournament. The higher cortisol values compared to baseline reflect with previous research (19-21), though comparisons should be carefully interpreted as they collected precompetition saliva samples 10-45 min before the onset of the competition, which is known to increase cortisol secretion as anticipation occurs (42).

Because cortisol manifests a circadian rhythm, peaking within the first hour of waking, it is hypothesised that the cortisol awakening response may represent forthcoming memory representations at waking, which assists with the orientation about the self in space and time, as well as the anticipation of the demands of the coming day (42). Factors that can influence the cortisol awakening response are sleep, menstrual cycle phase, contraceptive usage and a player's perception of stress (42). As mentioned, none of the players reported being either in their menstrual cycle phase or using any form of contraceptive, as both may result in higher cortisol secretion and negative mental states (20, 43).

In addition to anticipation of an event increasing cortisol secretion $(2,17,28)$ and somatic anxiety (27), the significantly higher values seen on day three might be explained by the importance of the forthcoming event. The outcome of this match determined the format and team to face for the forthcoming playoffs to enter the finals.

Our results demonstrated a significantly higher cortisol and anxiety response for the mornings prior to the matches lost, which is in accordance with previous research also reporting significant cortisol $(p=0.018)(27)$ and anxiety $(p<0.001)(23)$ increases prior to a losing match. They also reported a relationship $(r=0.4, p=$ $0.07)$ between anticipatory anxiety and cortisol levels (27) prior to a match lost, though these were not measured upon waking but $45 \mathrm{~min}$ before the competition. This is in contrast to the statement made by Bateup and colleagues (19), indicating that cortisol increases are unrelated to a competition outcome.

Due to no significant differences taking place between the ISP scores of the baseline data and day one of the tournament, the plausibility that the players might have been unfamiliar or confused with the testing procedure was ruled out. Therefore, making the baseline scores obtained valid to compare against the tournament data. As expected, a progressive increase in the TMD score took place over the course of the tournament, with the subscales fatigue rising and vigour decreasing from day two onwards. This might be due to increased anxiety levels, psychological stress, more difficult matches and opponents to face as well as accumulated fatigue taking place (44). The relationship observed between trait- and state-anxiety was previously reported $(5,39)$, indicating how players prone to anxiety tend to perceive a situation as more stressful and experience further performance decrements (5). In addition to this, the 
relationship observed between cortisol and stateanxiety is in accordance to previous studies (25, 40 ), though it should be carefully interpreted as the latter studies did not measure baseline values but only at $15-30 \mathrm{~min}$ preceding a competition. No other study could be found assessing whether a relationship exists between trait-anxiety and cortisol prior to a competition, and whether this psychological state can predict the underlying physiological mechanism. This might have implications for the development of a coaching program for collegiate soccer players as anxietyprone players might overanalyze an upcoming event, leading to performance decrements.

Our results did indeed indicate the value of implementing an anxiety questionnaire to predict underlying cortisol responses prior to a competition, and especially prior to a losing outcome. This can provide the coaching staff with the necessary information to prepare their players for the upcoming event. In addition, evaluating a player's proneness to anxiety prior to competition or during a training period, can offer the opportunity to implement the necessary coping strategies or mindset to overcome the stress experienced.

\section{CONCLUSION}

Though ample research is available on the hormonal and psychological effects of a competition on female athletes, only a few studies are available on the effect of multiple competitions played in a short time on baseline states. Furthermore, no research is available regarding African, and South African, female soccer players. This is the first study to evaluate the effect of a weeklong tournament on baseline cortisol and mental states and whether there are other plausible ways to determine the cortisol response to an upcoming event. We have confirmed how a tournament places great physical, physiological, and psychological stress on athletes, as seen with significant cortisol upsurges and negative mental states. Furthermore, due to the relationship seen between cortisol and the presence of anxiety, it is sensible to implement an anxiety questionnaire to determine and possibly predict, the cortisol response to stress. In addition, the coaching staff can implement an anxiety questionnaire to determine the innate anxiety states, as it might provide sensitive information on the psychological and physiological reaction to stress.

\section{APPLICABLE REMARKS}

- This study demonstrated the efficacy of implementing a psychological questionnaire aimed at determining the current and ongoing anxiety states, to predict plausible psychohormonal consequences on future sports performance. Measuring the innate anxiety states can provide valuable information on a player's proneness for stress, not only at a psychological level but also as seen with cortisol surges, which can have detrimental performance effects.

- A few limitations were however identified. Testing a larger population would prove valuable, not only for excluding outliers from slanting the statistics, but also to determine dissimilarities between starters, non-starters, positions, and duration of playtime and more profound correlations between variables. Furthermore, analyzing testosterone together with cortisol and the psychological questionnaires will provide valuable insights into the psycho-hormonal relationships that take place.

\section{ACKNOWLEDGEMENTS}

We thank the coaches and players of the respective soccer teams. We also thank the field personnel who were actively involved in the data capturing process.

\section{REFRENCES}

1. Chennaoui M, Bougard C, Drogou C, Langrume C, Miller C, Gomez-Merino D, et al. Stress Biomarkers, Mood States, and Sleep during a Major Competition: "Success" and "Failure" Athlete's Profile of HighLevel Swimmers. Front Physiol. 2016;7:94. doi: 10.3389/fphys.2016.00094 pmid: 27014092

2. Broodryk A, Pienaar C, Edwards D, Sparks M. Effects of a Soccer Tournament on the Psychohormonal States of Collegiate Female Players. J Strength Cond Res. 2019. doi: 10.1519/JSC.0000000000002993 pmid: 30817371

3. Martínez-Lagunas V, Niessen M, Hartmann U. Women's football: Player characteristics and demands of the game. J Sport Heal Sci. 2014;3(4):258-272. doi: 10.1016/j.jshs.2014.10.001

4. Strauss A, Pienaar C, Sparks M. The use of GPS analysis to quantify the internal and external match demands of semi-elite level female soccer players during a tournament. J Sport Sci Med. 2019;18:73-81. 
5. Horikawa M, Yagi A. The relationships among trait anxiety, state anxiety and the goal performance of penalty shoot-out by university soccer players. PLoS One. 2012;7(4):e35727. doi: 10.1371/journal.pone.0035727 pmid: 22539998

6. Eysenck MW, Derakshan N, Santos R, Calvo MG. Anxiety and cognitive performance: attentional control theory. Emotion. 2007;7(2):336-353. doi: 10.1037/1528-3542.7.2.336 pmid: 17516812

7. Radzi J, Yusuf S, Amir N, Mansor S. Relationship of pre-competition anxiety and cortisol response in individual and team sport athletes. In: Second International Conference on the Future of ASEAN (ICoFA). 2017.

8. Raglin JS, Morris MJ. Precompetition anxiety in women volleyball players: a test of ZOF theory in a team sport. Br J Sports Med. 1994;28(1):47-51. doi: 10.1136/bjsm.28.1.47 pmid: 8044494

9. Man F, Stuchlíková I, Kindlmann P. Trait-State anxiety, worry, emotionality, and self-confidence in toplevel soccer players. Sport Psychol. 2016;9(2):212-224. doi: 10.1123/tsp.9.2.212

10. Varzaneh AG, Saemi E, Shafinia P, Zarghami M, Ghamari A. Relationship between mental skills and anxiety interpretation in female volleyball players. Phys Cult Tour. 2011;18(1):19-24.

11. Martens R, Vealey RS, Burton D. Competitive anxiety in sport. 1st ed. Illinois: Human Kinetics; 1990.

12. Smyth J, Ockenfels MC, Porter L, Kirschbaum C, Hellhammer DH, Stone AA. Stressors and mood measured on a momentary basis are associated with salivary cortisol secretion. Psychoneuroendocrinol. 1998;23(4):353-370. doi: 10.1016/S0306-4530(98)00008-0

13. Nelson RJ. An introduction to behavioral endocrinology. 4th ed. Sunderland, Massachusetts: Sinauer Associates, Inc. Publishers2011.

14. Kudielka BM, Kirschbaum C. Awakening cortisol responses are influenced by health status and awakening time but not by menstrual cycle phase. Psychoneuroendocrinol. 2003;28(1):35-47. doi: 10.1016/S0306-4530(02)00008-2

15.Edwards DA, Casto KV. Women's intercollegiate athletic competition: cortisol, testosterone, and the dual-hormone hypothesis as it relates to status among teammates. Horm Behav. 2013;64(1):153-160. doi: 10.1016/j.yhbeh.2013.03.003 pmid: 23523743

16. Dickerson SS, Kemeny ME. Acute stressors and cortisol responses: a theoretical integration and synthesis of laboratory research. Psychol Bull. 2004;130(3):355-391. doi: 10.1037/0033-2909.130.3.355 pmid: 15122924

17. Casto KV, Edwards DA. Testosterone, cortisol, and human competition. Horm Behav. 2016;82:21-37. doi: 10.1016/j.yhbeh.2016.04.004 pmid: 27103058

18. Aizawa K, Nakahori C, Akimoto T, Kimura F, Hayashi K, Kono I. Changes of pituitary, adrenal and gonadal hormones during competition among female soccer players. J Sport Med Phys Fitness. 2006;46(2):322-327.

19. Bateup HS, Booth A, Shirtcliff EA, Granger DA. Testosterone, cortisol, and women's competition. Evol Hum Behav. 2002;23(3):181-192. doi: 10.1016/S1090-5138(01)00100-3

20. Casto KV, Edwards DA. Before, During, and After: How Phases of Competition Differentially Affect Testosterone, Cortisol, and Estradiol Levels in Women Athletes. Adapt Hum Behav Physiol [Internet]. 2016;2(1):11-25. doi: 10.1007/s40750-015-0028-2

21. Salvador A, Suay F, González-Bono E, Serrano MA. Anticipatory cortisol, testosterone and psychological responses to judo competition in young men. Psychoneuroendocrinol. 2003;28(3):364375. doi: 10.1016/S0306-4530(02)00028-8

22. Strahler K, Ehrlenspiel F, Heene M, Brand R. Competitive anxiety and cortisol awakening response in the week leading up to a competition. Psychol Sport Exerc [Internet]. 2010;11(2):148-154. doi: 10.1016/j.psychsport.2009.10.003

23. Oliveira T, Gouveia MJ, Oliveira RF. Testosterone responsiveness to winning and losing experiences in female soccer players. Psychoneuroendocrinology. 2009;34(7):1056-1064. doi: 10.1016/j.psyneuen.2009.02.006 pmid: 19278791

24.Edwards DA, Casto KV. Baseline cortisol moderates testosterone reactivity to women's intercollegiate athletic competition. Physiol Behav. 2015;142:48-51. doi: 10.1016/j.physbeh.2015.01.037 pmid: 25647361 
25.Haneishi K, Fry AC, Moore CA, Schilling BK, Li Y, Fry MD. Cortisol and stress responses during a game and practice in female collegiate soccer players. J Strength Cond Res. 2007;21(2):583-588. doi: 10.1519/R-20496.1 pmid: 17530979

26. Chida Y, Steptoe A. Cortisol awakening response and psychosocial factors: a systematic review and meta-analysis. Biol Psychol. 2009;80(3):265-278. doi: 10.1016/j.biopsycho.2008.10.004 pmid: 19022335

27. Aguilar R, Jimenez M, Alvero-Cruz JR. Testosterone, cortisol and anxiety in elite field hockey players. Physiol Behav. 2013;119:38-42. doi: 10.1016/j.physbeh.2013.05.043 pmid: 23743274

28. Filaire E, Dreux B, Massart A, Nourrit B, Rama LM, Teixeira A. Salivary alpha-amylase, cortisol and chromogranin A responses to a lecture: impact of sex. Eur J Appl Physiol. 2009;106(1):71-77. doi: 10.1007/s00421-009-0991-z pmid: 19190932

29. Salimetrics LLC, SalivaBio LLC. Saliva Collection and Handling Advice. In: Methods [Internet]. 3rd ed. State College, PA:Author2011.

30. Westermann J, Demir A, Herbst V. Determination of cortisol in saliva and serum by a luminesceneenhanced enzyme immunoassay. Clin Lab. 2004;50(1-2):11-24.

31. Kirschbaum C, Hellhammer DH. Salivary Cortisol. In: Encyclopedia of stress. 3rd ed. San Diego, CA: Academic Press, Inc2000.

32. Broodryk A, Pienaar C, Edwards D, Sparks M. The psycho-hormonal influence of anaerobic fatigue on semi-professional female soccer players. Physiol Behav. 2017;180:8-14. doi: 10.1016/j.physbeh.2017.07.031 pmid: 28782524

33. McNair DM, Lorr M, Droppleman LF. Profile of mood states. San Diego, CA: Educational and Industrial Testing Service1981.

34. Dean JE, Whelan JP, Meyers AW. An incredibly quick way to assess mood states: The incredibly short POMS. Annu Conf Assoc Adv Appl Sport Psychol. 1990.

35.Spielberger C, D. Manual for the State-Trait Anxiety Inventory STAI (Form Y) ("Self-Evaluation Questionnaire") [Internet].1983.

36. Barnes LLB, Harp D, Jung WS. Reliability Generalization of Scores on the Spielberger State-Trait Anxiety Inventory. Educ Psychol Meas. 2002;62(4):603-618. doi: 10.1177/0013164402062004005

37. Cohen J. Statistical Power Analysis for the Behavioral Sciences. 2nd ed. Erlbaum L, editor. Hillsday: New Jersey: Academic Press, Inc.1998.

38. Batterham AM, Hopkins WG. Making meaningful inferences about magnitudes. Int J Sports Physiol Perform. 2006;1(1):50-57. doi: 10.1123/ijspp.1.1.50

39.Ziomkiewicz A, Wichary S, Gomula A, Pawlowski B. Trait anxiety moderates the association between estradiol and dominance in women. Physiol Behav. 2015;143:97-103. doi: 10.1016/j.physbeh.2015.02.045 pmid: 25725118

40. Alix-Sy D, Le Scanff C, Filaire E. Psychophysiological responses in the pre-competition period in elite soccer players. J Sport Sci Med. 2008;7(4):446-454.

41.McGuigan MR, Egan AD, Foster C. Salivary Cortisol Responses and Perceived Exertion during High Intensity and Low Intensity Bouts of Resistance Exercise. J Sports Sci Med [Internet]. 2004;3(1):8-15.

42. Fries E, Dettenborn L, Kirschbaum C. The cortisol awakening response (CAR): facts and future directions. Int J Psychophysiol. 2009;72(1):67-73. doi: 10.1016/j.ijpsycho.2008.03.014 pmid: 18854200

43. Baker FC, Driver HS. Circadian rhythms, sleep, and the menstrual cycle. Sleep Med. 2007;8(6):613-622. doi: 10.1016/j.sleep.2006.09.011 pmid: 17383933

44. Mortatti AL, Moreira A, Aoki MS, Crewther BT, Castagna C, de Arruda AF, et al. Effect of competition on salivary cortisol, immunoglobulin A, and upper respiratory tract infections in elite young soccer players. J Strength Cond Res. 2012;26(5):1396-1401. doi: 10.1519/JSC.0b013e31822e7b63 pmid: 22516911 